\title{
Effects of thermal and mechanical activation methods on compressive strength of ordinary Portland cement-slag mortar
}

\author{
Fathollah Sajedi , Hashim Abdul Razak
}

Generally, the cement-slag mortars can be divided into three

groups as: ordinary Portland cement (OPC) mortars (OMs), Ordinary

Portland cement-slag mortars (OSMs), and slag mortars (SMs).

Based on other researches, it can be said that among the activation methods, Chemical $(C)$, Thermal $(T)$, and Mechanical $(M)$ are the most usual activation methods. However, for activation of the

OSMs, the combinational methods of activation such as ChemicalThermal (CT), Chemical-Mechanical (CM), Thermal-Mechanical (TM), and Chemical-Thermal-Mechanical (CTM) can be used. It is well known that for the mortars with cement particles like OMs and OSMs, the use of activation methods, including activators, are not effective [1,2], and then in this research work, only $T, M$, and TM methods were used. Not much research has been reported on the combinational activation methods of OSMs. The addition of alkalis to Portland cement results in a reduction of strength after 3 or 7 days, because hydration chemistry and the morphology of the hydration products are changed due to the presence of alkalis. OPC/slag mixes activated by alkalis showed a lower strength than the slag alone activated by an alkali [3].

Generally, it can be said that the SMs have both a low early and then ultimate strengths. The OMs have high early strengths and also relatively high ultimate strengths. Finally, the OSMs have low early and high ultimate strengths. However, some activation methods can be used to improve the strengths at early ages. Based on the results reported by other researches [4,5], it has been observed that by using chemical activators, early strength of the SMs can be improved.

Chemical activation refers to the use of some chemical substances

to activate the potential reactivity of cementitious components.

Some chemical activators are $\mathrm{Na}_{2} \mathrm{SiO}_{3}, \mathrm{NaOH}, \mathrm{KOH}$, and

$\mathrm{Na}_{2} \mathrm{CO}_{3}[6]$. The activator(s) can be added during the milling of cement/

slag or can be dissolved in the mixing water and added during the mixing of cement/slag pastes, mortars, and concrete. The technology is very simple and does not need extra equipment.

Raising temperature is more helpful to the reaction processes for higher reaction activation energies than those with lower reaction activation energies. Many researches [7] have shown that

Portland-cement slag has higher hydration activation energy than Portland cement and the higher the replacement of cement with slag, the higher the apparent hydration activation energy. Under normal conditions, blast-furnace slags show a higher reactivity 
with lime than other glassy pozzolans. Hardened cement pastes and concretes can reach their maximum strength within several hours through elevated temperature curing. However, the ultimate strength of hardened cement pastes and concretes has been shown to decrease with curing temperature. Other researchers have indicated that slag was more sensitive to heat than Portland cement due to its high apparent activation energy $[8,9]$. Roy and Idorn [8] even suggested that the combined effect of alkalis and heat may be synergetic during early hydration.

Heat curing is commonly used in the production of precast concrete elements to increase the rate of hydration and accelerate early age strength development. High elevated temperatures typically above 70 _ may lead to the deleterious expansion of concrete if it is subsequently exposed to a sufficient amount of moisture at ambient temperature. This expansion can cause the cracking of concrete, resulting in strength loss, a decreased service life or other serviceability and durability problems. Cracks and voids in damaged concrete are also often filled with ettringite [10]. Laboratory research demonstrates that many Portland cements can be made to expand if cured at sufficiently high temperatures and then subjected to moist environments. In practice, the most effective method of controlling expansion is to avoid exposure to high temperatures during curing for a prolonged period of time [10]. Curing temperature is the most important factor determining the potential for deleterious expansion of Portland cement products exposed to moist storage.

Prolonged grinding not only increases the surface area of a material, but it also increases the number of imperfections or active centers which exist at the edges, corners, projections, and places where the inter-atomic distances are abnormal or are embedded with foreign atoms. These centers are in a higher energy state than in the normal structure. The more such centers, the higher is the reactivity. If grinding is sufficiently prolonged, an upper limit of activity is attained; beyond which a continued increase in surface area does not produce any further increase in pozzolanic reactivity.

This paper considers the results of an investigation about OPC mortars and OPC-slag mortars which were studied after two curing regimes. These included water curing (wc) and curing at room temperature (ac). Five groups of mortars as: OM-ac and OSM/50-ac mixes (first group - G1), OM-wc and OSM/50-wc mixes (second group - G2), OM-T-ac and OSM/50-T-ac (third group - G3), OMMwc and OSM/50-M-wc mixes (fourth group - G4), and OMTMac and OSM/50-TM-ac mixes (fifth group - G5) were also used. Each group included two mixes; one was $\mathrm{OM}$ and the other was $\mathrm{OSM} / 50$ with the same conditions of casting and material properties. It was noted that groups 1, 3, and 5 were cured in room temperature and groups 2 and 4 cured in the water. However, it should 
be noted that the selection of curing regimes type depends on the strength results obtained from prior parts of the study [11,12].

The objective of the research is to study the compressive strength of OMs and OSMs/50 cured in room temperature and water regimes. Moreover, it is well known that OSMs/50 have low early strengths, and it is desired to improve by using the thermal, mechanical, and thermal-mechanical activation methods. Finally, the effects of the aforementioned activation methods are studied on the early and ultimate strengths of OMs and OSMs/50 and the results are compared. Also based on the results obtained, the optimum activation method is recommended. In addition, based on the initial and ultimate strengths view points, the best mix/group of the mortars is introduced. It is noted that in this case the loss of strength is an important factor.

2. Mix proportions, curing, and testing

Table 1 represents the mix proportions of the mixes. In all mixes $\mathrm{W} / \mathrm{B}=0.33, \mathrm{~S} / \mathrm{B}=2.25$ and silica sands were used. At first, based on particle size analysis (PSA), five groups of silica sands were mixed. Two min after that, cement was put into the mixture, followed by 2 min of mixing. Firstly, the mixing water was added to the mix and mixing was continued for two extra min, after which the required amount of super plasticizer was added. This mixing process was continued for $2 \mathrm{~min}$. Finally, the moulds were filled with two layers of fresh mortar. Each layer was then made compact with 10 impacts by using a rod of $16 \mathrm{~mm}$ in diameter. Twenty-four hours after casting, the specimens were demoulded and cured in room temperature with $28 \pm 4 \_\mathrm{C}$ and $65 \pm 20 \%$ relative humidity $(\mathrm{RH})$ or in water $23 \pm 2 \_C$ with time needed for breaking the specimens

\subsection{Materials}

The properties of the materials that have been used in the study are described as follows:

\subsubsection{Cement}

The cement used in all mixes was OPC. ASTM C109/C109M-08

[13] was used for the determination of the compressive strength of hydraulic cement mortars by using $50 \mathrm{~mm}$ side cubes as specimens.

The specific gravity of cement used is about 3.14. Based on particle size analysis (PSA) tests, the specific surface area (SSA) for OPC was determined to be $1893.9 \mathrm{~m} 2 / \mathrm{kg}$. The PSA diagram for the cement used in the study without use of grinding and with the use of duration of $6 \mathrm{~h}$ grinding is shown in Figs. 1 and 2. The chemical compositions of OPC used in this research have been determined by the X-ray fluorescence spectrometer (XRF) test. The results are given in Table 2. 


\subsubsection{Slag}

The specific gravity GGBFS is approximately 2.87 , with its bulk density varying in the range of $1180-1250 \mathrm{~kg} / \mathrm{m} 3$. The color of the slag is normally whitish (off-white). Based on the PSA test, SSA for slag has been determined at $3597.2 \mathrm{~m} / \mathrm{kg}$. It can be seen that the SSAslag $=1.90 \_$SSAOPC, which means that particles of slag are $90 \%$ finer than those of OPC. The compositions of slag are given in Table 2. As with all cementing materials, the reactivity of the slag is determined by its SSA. The PSA diagram for the slag used in the study without use of grinding and with use of duration of $6 \mathrm{~h}$ grinding is shown in Figs. 1 and 2.

In general, increased fineness results in better strength development, but in practice, fineness is limited by economic and performance considerations, and factors such as setting time and shrinkage. For better performance, the fineness of slag must be greater than that of cement. Based on the definition of the slag Full text available at :

http://www.sciencedirect.com/science/article/pii/S026130691000525X

http://ac.els-cdn.com/S026130691000525X/1-s2.0-S026130691000525X-main.pdf? tid=1229bb4a-7820-11e38e47-00000aacb361\&acdnat $=1389156669$ c78b476f13a6d3bb40a13b23d78b741e 